Onkologe 2015 $21: 55-56$

DOI 10.1007/s00761-014-2854-4

Online publiziert: 14. Januar 2015

(c) Springer-Verlag Berlin Heidelberg 2015

S. Singer ${ }^{1}$ U. Goerling ${ }^{2}$ J. Weis ${ }^{3}$ A. Schumacher ${ }^{4}$ B. Koch ${ }^{5}$. P.M. Schlag ${ }^{6}$

${ }^{1}$ Abt. Epidemiologie und Versorgungsforschung, Institut für Medizinische Biometrie, Epidemiologie und Informatik, Universitätsmedizin der Johannes-Gutenberg-Universität Mainz

2 Bereich Psychoonkologie, Charité Comprehensive Cancer Center, Universitätsmedizin Berlin

${ }^{3}$ Abt. Psychoonkologie, Klinik für Tumorbiologie, Freiburg

${ }^{4}$ Medizinische Klinik A (Hämatologie und Onkologie), Universitätsklinikum Münster

${ }^{5}$ Springer Medizin, Heidelberg

${ }^{6}$ Berlin

\title{
Neue Rubrik Psychoonkologie
}

\section{Integraler Bestandteil des onkologischen Themenspektrums}

Wie bei kaum einer anderen Erkrankung werden die behandelnden Ärzte in der Versorgung von Tumorpatienten mit den besonderen Ängsten und Sorgen ihrer Patienten, mit Verzweiflung, tiefer Trauer, Hilflosigkeit und auch Wut konfrontiert. Manche Ärzte weichen der Auseinandersetzung mit diesen Themen eher aus, manche sehen sie als integralen Teil ihrer Tätigkeit an und versuchen, die Patienten auch auf dieser Ebene zu unterstützen, vielleicht mitunter bis über die Grenze der eigenen Belastbarkeit hinaus. Manche Ärzte wissen oft auch nicht recht, wie sie mit dieser Problematik umgehen können oder wie sie professionelle Unterstützung und Orientierung finden.

Die Psychoonkologie hat sich mittlerweile als ein eigenes Arbeitsgebiet in der Onkologie etabliert und kann auf der Basis langer, klinischer Erfahrung sowie wissenschaftlicher Forschungsergebnisse wichtige Hilfestellungen nicht nur für die Patienten und deren Angehörige, sondern auch für die behandelnden Ärzte geben.

Herausgeber und Verlag sind daher sehr dankbar, ein engagiertes Team von ausgewiesenen Psychoonkologen gefunden zu haben, die Der Onkologe regelmäßig mit dieser Thematik bereichern werden.

Vor diesem Hintergrund eröffnen wir eine neue Rubrik mit dem Titel „Psychoonkologie“. Mit Beginn der vorliegenden Ausgabe werden wir kontinuierlich darstellen, wie die psychosoziale Betreuung von Tumorpatienten nach aktuellen Erkenntnissen inhaltlich gestaltet und praktisch weiter verbessert werden kann. Dazu gehört beispielsweise, dass Sie erfahren, was konkret ein Psychoonkologe tut, wenn er mit Ihren Patienten spricht - das betrifft sowohl Diagnostik als auch Interventionen. Dabei soll auch erörtert werden, wodurch seelische Probleme infolge von Krankheit und Behandlung entstehen bzw. gelindert werden können und welche Rolle die Beziehung zum Arzt oder die Biographie des Patienten dabei spielen. Wir werden auch zeigen, wie Ärzte ihre kommunikative Kompetenz in den Gesprächen mit Patienten und Angehörigen verbessern können.

Darüber hinaus möchten wir Ihnen regelmäßig aktuelle Studienergebnisse zu psychoonkologischen Forschungsbereichen wie Lebensqualität, Fatigue und psychischen Belastungen bei Krebs präsentieren und ihre Bedeutung für die klinische Praxis einordnen.

D) Es geht nicht darum, dem Körper weniger, sondern der Seele mehr Aufmerksamkeit zu widmen

Gemäß dem interdisziplinären Charakter von Der Onkologe sollen die psychoonkologischen Themen nach Möglichkeit dem jeweiligen Leitthema einer Ausgabe zugeordnet sein. Dies soll auch dazu bei- 
Fachnachrichten

tragen, die Integration der medizinischen und psychoonkologischen Betreuung zu optimieren und die Zusammenarbeit in einem interdisziplinären Team zu stärken. Uns geht es nicht darum, dem Körper weniger, sondern der Seele mehr Aufmerksamkeit zu widmen.

Wir freuen uns auf Sie als interessierte und kritische Leserinnen und Leser, deren spezielle Anfragen wir stets gerne beantworten werden.

Ihre Rubrikherausgeber

Susame Gilys

Susanne Singer

Un falizy

Ute Goerling

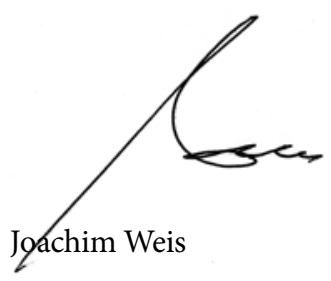

Andrea Ychumcher

Andrea Schumacher

Für den Verlag

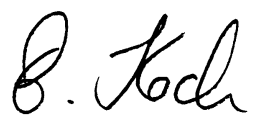

Bettina Koch

Für die Herausgeber

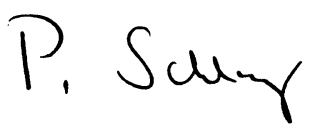

Peter M. Schlag
Korrespondenzadresse

Prof. Dr. S. Singer

Abt. Epidemiologie und Versorgungsforschung, Institut für Medizinische Biometrie, Epidemiologie und Informatik, Universitätsmedizin der Johannes-GutenbergUniversität Mainz

Obere Zahlbacher Straße 69, 55131 Mainz singers@uni-mainz.de

Einhaltung ethischer Richtlinien

Interessenkonflikt. S. Singer, U. Goerling, J. Weis, A. Schumacher, B. Koch und P.M. Schlag geben an, dass kein Interessenkonflikt besteht.
Fokus USA: ONKO-Internetportal berichtet von den Kongressen ASH und SABCS

Mit Video-Expertenrunden, Live-Interviews und Kongressberichte vom Jahrestreffen der American Society of Hematology (ASH) und dem San Antonio Breast Cancer Symposiums (SABCS) startet das ONKO-Internetportal in das Jahr 2015. Mehr als 20.000 Besucher aus aller Welt kamen im Dezember 2014 zum ASH-Kongress nach San Francisco, um aktuelle Forschungsdaten zu diskutieren. Beim Thema Lymphome erweckten insbesondere Ergebnisse zur Antikörpertherapie bei Morbus Hodgkin große Aufmerksamkeit; beim Multiplen Myelom waren neue Biomarker und Stammzelltransplantation wichtige Themen. Im Fokus der Expertenrunde Leukämien des ONKOInternetportals stehen diesmal molekulare Charakterisierung und innovative Therapien, beispielweise mit chimären Antikörpern, den sogenannten "Killerzellen“. Fast zeitgleich wurden auf dem weltweit größten Brustkrebskongress SABCS eine Vielzahl neuer Studienergebnisse vorgestellt. Im Mittelpunkt der Expertendiskussionen standen vor allem die IBIS-I-Studie zur Chemoprävention mit Tamoxifen, die SOFT-Studie zum Vergleich einer adjuvanten endokrinen Therapie mit und ohne Ovarsuppression, die GeparSepto-Studie zur neoadjuvanten Therapie mit nab-Paclitaxel sowie die deutsche BMBC-Studie zu Hirnmetastasen bei Brustkrebs. Im Rahmen der ONKO-Expertenrunde Mammakarzinom geben deutsche Experten ihre Einschätzung zur Relevanz der Ergebnisse für die klinische Praxis. Alle Interviews, Expertenrunden und Kongressberichte sind unter www.krebsgesellschaft.de/kongresse online abrufbar.

Quelle: Deutsche Krebsgesellschaft e.V. (DKG), www.krebsgesellschaft.de

56

Der Onkologe $1 \cdot 2015$ 